\title{
A Re-Evaluation of the Morphology of a Bicontinuous Block Copolymer-Ceramic Material
}

\author{
Gilman E. S. Toombes, ${ }^{\dagger}$ Adam C. Finnefrock, ${ }^{\dagger, *}$ Mark W. Tate, ${ }^{\dagger}$ Ralph Ulrich,,$* \perp$ \\ Ulrich Wiesner, ${ }^{\S}$ and Sol M. Gruner*, ${ }^{*}, \|$ \\ Department of Physics, Department of Materials Science and Engineering, and Cornell High Energy \\ Synchrotron Source (CHESS), Cornell University, Ithaca, New York 14853, and Max-Planck-Institute \\ for Polymer Research, Postfach 3148, D-55021 Mainz, Germany
}

Received July 11, 2007; Revised Manuscript Received September 7, 2007

\begin{abstract}
The structures of a poly(isoprene-block-ethylene oxide) (PI- $b$-PEO) block copolymer-directed aluminosilicate mesostructure and the resulting ceramic material obtained from calcination were studied via smallangle X-ray scattering (SAXS) and transmission electron microscopy (TEM). The PI minority phase (volume fraction 0.36 ) formed a continuous network of channels, previously reported ${ }^{1,2}$ to be consistent with the plumber's nightmare ${ }^{3}$ morphology. The solvent casting process used to form the material caused it to shrink uniaxially by $\sim 30 \%$, deforming the network structure within it. Calculated structure factors for constant-curvature and constantthickness models of a distorted double gyroid structure are consistent with SAXS from the material, while [100] and [111] projections of the distorted double gyroid structure match the TEM data. Because the structural data from the material is most consistent with a distorted version of the double gyroid morphology, the previous assignment of the plumber's nightmare morphology must be reconsidered. Approaches for structural assignment are also discussed.
\end{abstract}

\section{Introduction}

A number of bicontinuous network structures have been identified in soft-condensed matter systems including the double gyroid $^{4,5}(\mathrm{G})$, double diamond ${ }^{6,7}(\mathrm{D})$, plumber's nightmare ${ }^{3,6}$ $(\mathrm{P})$, and I-WP ${ }^{4,8}$ morphologies illustrated in Figure 1. Identification of these complex structures can be challenging, ${ }^{9}$ and a combination of characterization techniques is frequently required. Many bicontinuous materials lack long-range periodicity ${ }^{10,11}$ and single-crystal specimens are certainly the exception. ${ }^{9}$ Space-group assignment is difficult when dynamic and static disorder smear out all but a handful of diffraction peaks. ${ }^{2,9}$ Furthermore, some 2-D projections of different network structures are quite similar in appearance. ${ }^{12-14}$ Consequently, in some instances further study of a material has led to a revision of an earlier structural assignment. ${ }^{12,15,16}$

This report describes such a re-evaluation for a solvent-cast poly(isoprene-block-ethylene oxide) (PI- $b$-PEO) copolymer/ aluminosilicate composite material and the resulting ceramic obtained from calcination. The thickness of the solvent-cast film was approximately $0.5-1.0 \mathrm{~mm}$ while the volume fraction of the PI minority phase was 0.36 . In an earlier study, ${ }^{1,2}$ some of us reported the material's structure to be consistent with the plumber's nightmare $(\mathrm{P})$ morphology and excluded the double gyroid (G) structure because small-angle X-ray scattering (SAXS) from the material showed $\{110\}$ and $\{200\}$ Bragg reflections forbidden by the symmetries of the double gyroid structure $\left(\mathrm{G}\right.$, space group $\left.\operatorname{Ia} \underline{3} d, \mathrm{Q}^{230}\right) .{ }^{17}$ However, the solventcasting process uniaxially compressed the film along the film normal by $\sim 30 \%$, as illustrated in Figure 2. Compression of a cubic lattice breaks the screw-axis and glide-plane symmetries

* Corresponding author. E-mail: smg26@cornell.edu.

$\dagger$ Department of Physics.

$\doteqdot$ Present addresses: Merck Research Laboratories, WYN-2, 466 Devon Park Drive, Wayne, PA 19087 (A.C.F.); Lanxess Deutschland GmbH, Gebäude G17, 41539 Dormagen, Germany (R.U.)

$\S$ Department of Materials Science and Engineering

"Cornell High Energy Synchrotron Source (CHESS).

${ }^{\perp}$ Max-Planck-Institute for Polymer Research.
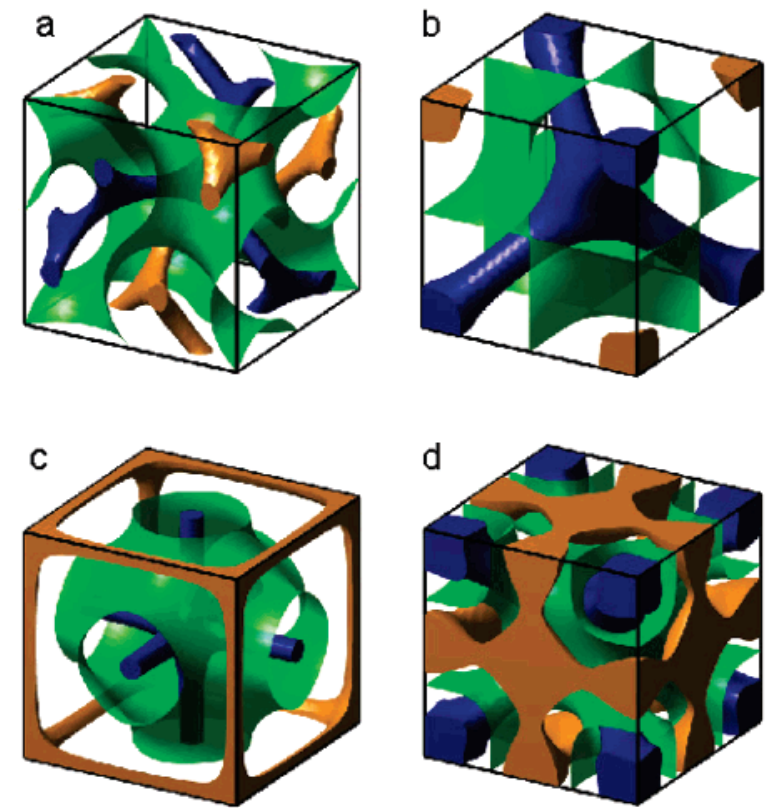

Figure 1. Unit cells of the (a) double gyroid (G) structure, space group Ia $3 d, \mathrm{Q}^{230}$; (b) double diamond (D) structure, $P n 3 m, \mathrm{Q}^{224}$; (c) plumber's nightmare (P) structure, $\operatorname{Im} 3 m, \mathrm{Q}^{229}$; and (d) I-WP network structure, $\operatorname{Im} 3 m, \mathrm{Q}^{229}$. The green IPMS divides space between the gold and blue skeletal frames, each of which forms a continuous network in all three spatial directions. Skeletal frames and IPMS were calculated using the level-set approximation. ${ }^{48}$

of the Ia $3 d$ space-group and $\{110\}$ and $\{200\}$ reflections are no longer forbidden. ${ }^{18,19}$ Thus, the observation of $\{110\}$ and $\{200\}$ reflections does not rigorously exclude a double gyroid structure distorted by lattice compression (distorted-G).

To determine if a distorted double gyroid (distorted-G) structure was consistent with the experimental data, the structural deformations caused by lattice contraction were calculated for models of the double gyroid structure with elastic $\left(\mathrm{G}_{\mathrm{el}}\right)$, constantthickness $^{20}\left(\mathrm{G}_{\mathrm{CT}}\right)$, and constant-curvature ${ }^{20}\left(\mathrm{G}_{\mathrm{CC}}\right)$ material properties. Structure factors for these three distorted double 
a

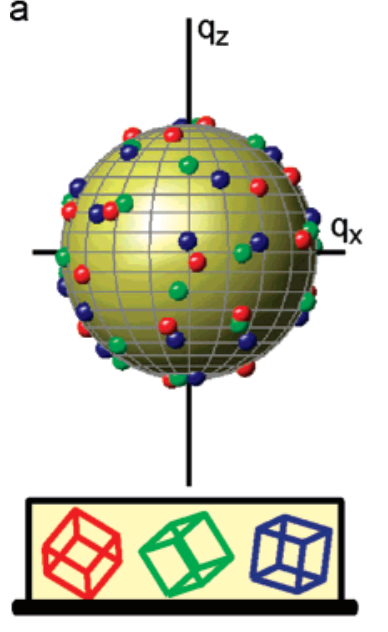

C

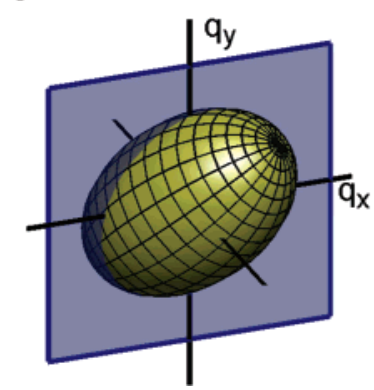

b
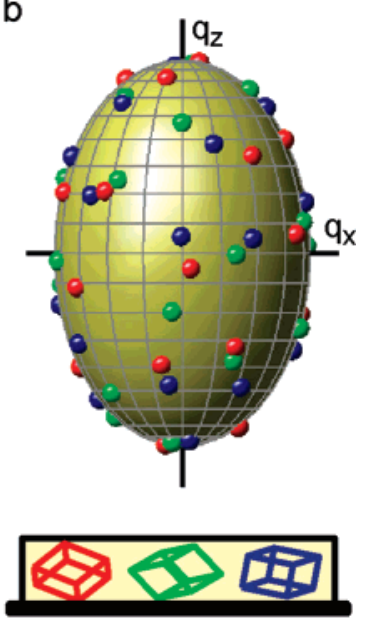

d

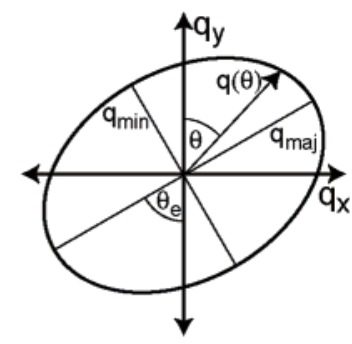

Figure 2. Effect of sample contraction on reciprocal space structure. (a) Ideal isotropic case. The orientation of three crystallites in a film is illustrated below, while the position of the corresponding $\{211\}$ reflections in reciprocal space is marked above in the same color. For a given value of $h^{2}+k^{2}+l^{2}$, all reflections lie on the surface of a sphere. (b) Following uniaxial contraction of the film, the unit cell of individual crystallites also contracts along the film normal. The reciprocal lattice becomes elongated and reflections with a given value of $h^{2}+k^{2}+l^{2}$ lie on the surface of a prolate spheroid, as shown for the $\{211\}$ reflections. (c) Intersection of the Ewald sphere and the prolate spheroid of the $\{h k l\}$ Bragg reflections. (d) Elliptical shape of the resulting diffraction rings.

gyroid models were calculated and the intensity for $\{110\}$, $\{200\}$, and other reflections agree well with SAXS from the material. [100] and [111] projections of a distorted double gyroid structure were also calculated and resemble transmission electron microscopy (TEM) micrographs from the material. Thus, a distorted double gyroid structure is consistent with the structural data from the material, and the previous assignment of a plumber's nightmare $(\mathrm{P})$ morphology must be re-evaluated.

\section{Results}

Experimental Structure Factors. As reported earlier, ${ }^{2}$ SAXS data was gathered from both the initial PI- $b$-PEO/aluminosilicate films (as-made) and from mesoporous aluminosilicate (calcined) produced by calcining these films. A representative 2-D SAXS pattern is shown in Figure 3a. Distinct Bragg spots are seen in Figure $3 \mathrm{a}$ because the crystallites were quite $\operatorname{large}^{1}$ and so for any given orientation of the sample, only a small number $(\sim 5)$ of crystallites satisfied the Bragg diffraction condition. As shown in Figure 2, uniaxial contraction of the film during the solvent casting process caused the Bragg spots to lie on ellipses rather than circles. ${ }^{2,21}$ These ellipses would be smeared out by a conventional azimuthal powder average so structure factors must be estimated by other techniques. For an individual image, the intensity of individual Bragg spots were estimated by fitting
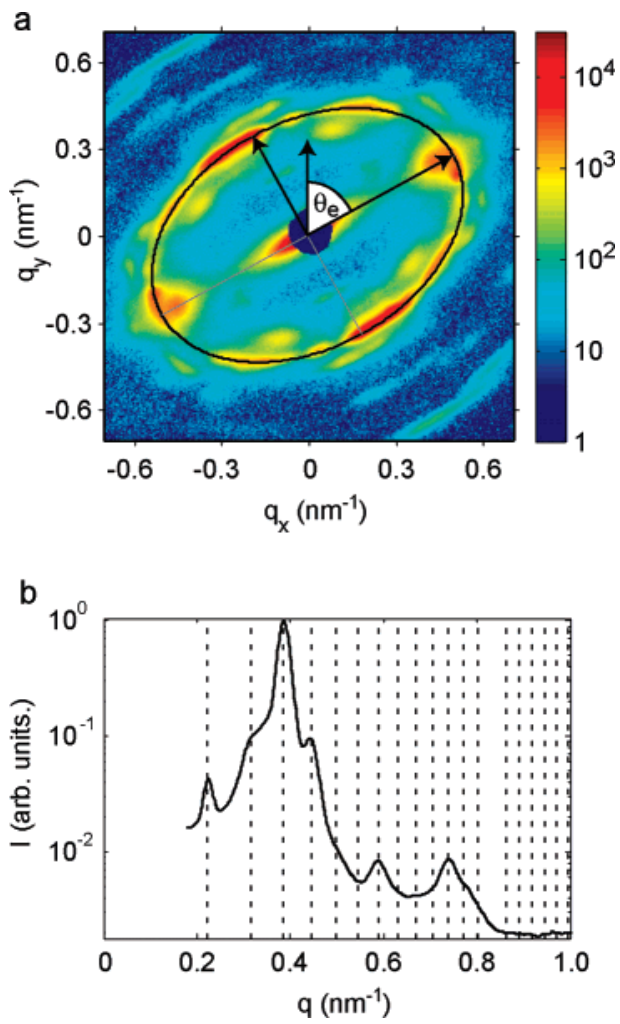

Figure 3. (a) Representative 2-D SAXS pattern (calcined material). The bright $\{211\}$ Bragg reflections lie on the ellipse marked in black. The ellipse radii are $q_{\text {maj }}=0.572 \pm 0.007 \mathrm{~nm}^{-1}$ and $q_{\text {min }}=0.390 \pm$ $0.005 \mathrm{~nm}^{-1}$ while the angle between the sample rotation axis (vertical) and the semi-major axis is $\theta_{\mathrm{e}}=61.3^{\circ} \pm 2.5^{\circ}$. (b) Pseudo-powder average obtained by integration of a rotation series as described in the text (calcined material). The dotted lines indicate even values of $h^{2}+k^{2}+$ $l^{2}$ for a cubic lattice with unit cell size of $d=39.2 \pm 0.8 \mathrm{~nm}$.

the surrounding scattering intensity, $I(q, \theta)$, to a Gaussian peak profile of the form

$$
\begin{aligned}
I(q, \theta)=B+\frac{I_{0}}{(2 \pi)^{3 / 2} \theta_{\mathrm{w}}{ }^{2}\left(q_{\mathrm{w}}{ }^{2}+q_{0}{ }^{2}\right) q_{\mathrm{w}}} \times \\
\exp \left(\frac{-\left(q-q_{0}\right)^{2}}{2 q_{\mathrm{w}}{ }^{2}}+\frac{-\left(\theta-\theta_{0}\right)^{2}}{2 \theta_{\mathrm{w}}{ }^{2}}\right)
\end{aligned}
$$

where $I_{0}$ is the integrated scattering intensity, $q_{0}$ and $\theta_{0}$ are the radial and angular position of the peak center, $q_{\mathrm{w}}$ and $\theta_{\mathrm{w}}$ are the radial and angular width of the peak, and $B$ accounts for the background scattering level. Structure factors were roughly estimated by averaging the intensity per spot for each set of reflections, $\{h k l\}$, and the results of this procedure are reported in Table 1. However, the small number of crystallites, differences in the size of individual crystallites, and differences in how close each crystallite is to the Bragg diffraction condition make these estimates quite imprecise.

These problems were addressed using a rotation series in which the sample was rotated to different angles about an axis perpendicular to the incident beam and SAXS images were taken at each position. To combine data taken at different rotation angles, the effect of rotation on the elliptical scattering ring shape was accounted for. Figure 2 illustrates how contraction along an axis in real space causes an elongation along the same axis in reciprocal space so the $\{h k l\}$ lattice vectors lie on the surface of a prolate spheroid. ${ }^{2}$ On a 2-D SAXS pattern, the observed $\{h \mathrm{kl}\}$ reflections are located at the intersection of this spheroid with the Ewald sphere as shown in Figure 2c,d. 
Table 1. Experimental Structure Factors $\left|S_{h k l}\right|^{2}$

\begin{tabular}{cllllll}
\hline & & \multicolumn{2}{c}{ as-made } & & \multicolumn{2}{c}{ calcined $^{n} h^{2}+k^{2}+l^{2}$} \\
\cline { 6 - 7 } \cline { 5 - 6 } & $\{h k l\}$ & spots $^{a}$ & powder $^{b}$ & & spots $^{a}$ & powder $^{b}$ \\
\hline 2 & 110 & $0.4(6)$ & $0.3 \pm 0.1$ & & $1.3(6)$ & $1.5 \pm 0.1$ \\
4 & 200 & $8.0(2)$ & $56 \pm 2$ & & $6.1(2)$ & $28 \pm 1$ \\
6 & 211 & $100(14)$ & 100 & & $100(22)$ & 100 \\
8 & 220 & $16(6)$ & $8.1 \pm 0.7$ & $12(6)$ & $17 \pm 1$ \\
10 & 310 & nd (0) & 0.0 & nd (0) & 0.0 \\
12 & 222 & nd (0) & 0.0 & nd (0) & 0.0 \\
14 & 321 & $1.6(8)$ & nd & $3.2(8)$ & $0.7 \pm 0.1$ \\
16 & 400 & $1.0(2)$ & nd & $3.0(2)$ & $1.8 \pm 0.4$ \\
18 & $330 / 411$ & nd (0) & nd & nd (0) & $0.34 \pm 0.06$ \\
20 & 420 & nd (0) & nd & $2.7(4)$ & $0.7 \pm 0.1$ \\
22 & 332 & $0.9(10)$ & nd & & $4.7(12)$ & $2.8 \pm 0.4$ \\
24 & 422 & $0.3(2)$ & nd & & $1.7(4)$ & $1.3 \pm 0.1$ \\
26 & 431 & nd (0) & nd & & $1.2(2)$ & $0.29 \pm 0.04$
\end{tabular}

${ }^{a}$ Average Bragg spot intensity normalized to $\{211\}$ reflections. The number of spots for each $\{h k l\}$ is given in parentheses. For some $\{h k l\}$, no reflections were observed as indicated by the letters "nd". ${ }^{b}$ Fit to "pseudopowder" average of scattering intensity made by summing scattering from a rotation series. The quoted errors are for the nonlinear least-squares fit and do not include systematic effects. The lower signal-to-noise for the uncalcined sample meant that reflections with large $h^{2}+k^{2}+l^{2}>12$ could not be reliably distinguished above the background scattering level as indicated by the letters "nd".

The elliptical shape of the scattering ring is related to the contraction of the sample by

$$
\begin{gathered}
\epsilon\left(\phi_{n}\right)=\sqrt{1-\left(\frac{q_{\text {min }}}{q_{\text {maj }}}\right)^{2}}=\sqrt{\left(2 s-s^{2}\right)\left(1-\sin ^{2}\left(\theta_{n}\right) \sin ^{2}\left(\phi_{n}\right)\right)} \\
\theta_{\mathrm{e}}\left(\phi_{n}\right)=\arctan \left(\tan \left(\theta_{n}\right) \cos \left(\phi_{n}\right)\right)
\end{gathered}
$$

where $\epsilon$ is the ellipse eccentricity, $\theta_{\mathrm{e}}$ is the angle between the $y$-axis and the ellipse semi-major axis, $q_{\min }$ and $q_{\max }$ are the semi-minor and semi-major ellipse radii, $s$ is the fractional contraction of the film, $\theta_{n}$ is the angle between the contraction axis and the $y$-axis, and $\phi_{n}$ is the angle between the $x$-axis and the projection of the contraction axis in the $x-z$ plane. The scattering ring has the greatest eccentricity when the X-ray beam is perpendicular to the sample normal $\left(\theta_{n}=0^{\circ}\right.$ and/or $\left.\phi_{n}=0^{\circ}\right)$ and is circular when the X-ray beam is parallel to the sample normal $\left(\theta_{n}=\phi_{n}=90^{\circ}\right)$. As described previously, ${ }^{2}$ these equations were fitted to the shape of scattering rings in a rotation series to determine the size of the unit cell and magnitude of uniaxial contraction. The as-made material had a unit cell of $d$ $=63.3 \pm 1.0 \mathrm{~nm}$ and a contraction of $s=30 \% \pm 3 \%$. Following calcination, the unit cell reduced to $d=39.2 \pm 0.8$ $\mathrm{nm}$ while the contraction was $s=33.7 \% \pm 1.3 \%$. The mean value of the $\{h k l\}$ reflections was calculated by averaging the scattering intensity on the surface of prolate spheroidal shells. The result of this process was a pseudo-powder average

$$
\begin{gathered}
I(q)=\int_{\theta, \phi} I(q g(\theta, \phi), \theta, \phi) \times \frac{g^{3}(\theta, \phi)(1-s) \sin \theta d \theta d \phi}{4 \pi} \\
g(\theta, \phi)=\left(1-\epsilon^{2}(\phi) \cos ^{2}\left(\phi-\theta_{\mathrm{e}}(\phi)\right)\right)^{-1 / 2}
\end{gathered}
$$

where the average was taken over the surface of a spheroid rather than a sphere. Rotation series were used to evaluate $I(q)$, and a plot for the calcined material is shown in Figure $3 \mathrm{~b}$. The intensity of scattering peaks was determined by fitting the 1-D scattering profile via nonlinear least-squares to the form,

$$
\begin{array}{r}
I(q)=\sum_{j=2,4,6 \ldots} \frac{I_{j} m_{j}}{j} V\left(q d-2 \pi j^{1 / 2}, \sigma_{\mathrm{L}}(j), \sigma_{G}(j)\right)+\sum_{k=0}^{3} \frac{B_{k}}{q^{k}} \\
\sigma_{\mathrm{L}}(j)=\sqrt{\sigma_{L_{0}}^{2}+\sigma_{L 1}^{2} j}, \sigma_{G}(j)=\sqrt{\sigma_{G 0}^{2}+\sigma_{G 1}^{2} j}
\end{array}
$$

where $I_{j}$ and $m_{j}$ are the intensity and multiplicity of the $j=h^{2}$ $+k^{2}+l^{2}$ scattering peak, $V$ is the Voigt function, ${ }^{22,23} \sigma_{\mathrm{L} 0}, \sigma_{\mathrm{L} 1}$, $\sigma_{\mathrm{G} 0}$, and $\sigma_{\mathrm{G} 1}$ describe the width of each scattering peak, and $B_{k}$ are coefficients describing background scatter. The resulting peak intensities for as-made and calcined materials are reported in Table 1. The $\{200\}$ reflections show the largest difference between the pseudo-powder average and Bragg spot estimates, but this is not surprising given the small number of Bragg spots $(N=2)$.

For both the as-made and calcined material, the $\{211\}$ reflections have the greatest intensity followed by the intermediate intensity of the $\{220\}$ and $\{200\}$ reflections while the $\{110\}$ reflections are relatively weak.

Model Structure Factors. The double gyroid, ${ }^{24}$ double diamond, ${ }^{7}$ and $\mathrm{I}^{-\mathrm{WP}^{25}}$ structures have all previously been observed in bicontinuous silica-type materials. Table 2 reports structure factors for double gyroid (G), double diamond (D), plumber's nightmare $(\mathrm{P})$, and I-WP structures calculated using the parametric equations of Garstecki and Holyst. ${ }^{26}$ There are distinct differences between the structure factors of the material and each of these models.

The double diamond (D) structure (Figure 1b) has strong $\{110\}$ and $\{111\}$ reflections and weak $\{211\}$ reflections. As the material had weak $\{110\}$ reflections, no $\{111\}$ reflections and strong $\{211\}$ reflections, the double diamond morphology is quite unlikely. The main discrepancies for the I-WP structure (Figure 1d) are its weak $\{211\}$ reflections, strong $\{110\}$ reflections, and relatively strong $\{310\}$ and $\{222\}$ reflections. The structure factors in Table 2 are for the most likely I-WP structure in which the minority PI phase (volume fraction 36\%) occupies the network of 4-way nodes (gold). Structures with the minority phase in the network of 8-way nodes (blue), both networks, or the matrix show no better agreement. ${ }^{27}$ The plumber's nightmare $(\mathrm{P})$ structure (Figure 1c) has strong $\{110\}$ reflections because the 6-way nodes are located at the corner and center of the unit cell. Thus, the plumber's nightmare morphology also has difficulty accounting for the experimental data because the observed $\{110\}$ reflections were weak. Finally, a cubic double gyroid (G) structure (Figure 1a) cannot account for the observed $\{110\}$ and $\{211\}$ reflections as these are forbidden by the symmetries of the Ia $3 \mathrm{~d}$ space group. ${ }^{17}$

Comparison to these cubic structures can be misleading, though, because the crystallites in the material were triclinic. Each triclinic crystallite was related to a cubic lattice by a uniaxial contraction/expansion, but the compressed unit cell could not have all of the crystallographic symmetries allowed in a cubic unit cell. ${ }^{18,19}$ However, depending on how the contraction affected the structure inside the unit cell, the triclinic crystallites could still have the same structure factors as a symmetric, cubic structure. This effect is illustrated in Figure 4 for a 2-D structure with a 4-fold rotational symmetry axis. If the gray and white domains compress proportionately (linear/ affine transformation) as shown in Figure $4 b$, the structure factors are effectively unchanged, even though the rotational symmetry is broken by the unit cell contraction. In contrast, the unit cells in parts $c$ and $d$ of Figure 4 have different structure factors because the white and gray domains have changed shape relative to the lattice. Thus, a contracted or stretched lattice only 
Table 2. Structure Factors $\left|S_{h k l}\right|^{2}$ for Model Network Structures

\begin{tabular}{|c|c|c|c|c|c|c|c|c|c|}
\hline$h^{2}+k^{2}+l^{2}$ & $\mathrm{D}^{a, b}$ & $\mathrm{P}^{a}$ & $\mathrm{I}-\mathrm{WP}^{a}$ & $\mathrm{G}^{a}$ & $\mathrm{G}_{\mathrm{el}}^{c, d}$ & $\mathrm{G}_{\mathrm{CC}}^{e, d}$ & $\mathrm{G}_{\mathrm{CI}}^{f, d}$ & as-made $^{g}$ & calcined $^{g}$ \\
\hline 2 & 100 & 100 & 42 & - & 0.43 & 28 & 23 & $0.3 \pm 0.1$ & $1.5 \pm 0.1$ \\
\hline 3 & 71 & $-h$ & - & - & - & - & - & 0.0 & 0.0 \\
\hline 4 & 6.5 & 89 & 100 & - & 1.0 & 95 & 96 & $56 \pm 2$ & $28 \pm 1$ \\
\hline 6 & 1.3 & 22 & 1.4 & 100 & 100 & 100 & 100 & 100 & 100 \\
\hline 8 & 0.0 & 0.0 & 5.3 & 38 & 41 & 26 & 77 & $8.1 \pm 0.7$ & $17 \pm 1$ \\
\hline 9 & 0.31 & - & - & - & - & - & - & 0 & 0.0 \\
\hline 10 & 0.47 & 0.05 & 8.6 & - & 0.08 & 1.2 & 7.6 & 0 & 0.0 \\
\hline 12 & 3.0 & 0.06 & 2.5 & - & 0.0 & 0.1 & 0.9 & 0 & 0.0 \\
\hline 14 & 0.48 & 1.4 & 0.18 & 0.21 & 0.21 & 0.9 & 3.2 & nd & $0.7 \pm 0.1$ \\
\hline 16 & 0.0 & 1.1 & 0.62 & 0.95 & 0.92 & 5.0 & 2.4 & nd & $1.8 \pm 0.4$ \\
\hline 17 & 2.1 & - & - & - & - & - & - & nd & 0.0 \\
\hline 18 & 0.57 & 1.95 & 0.45 & & 0.08 & 2.0 & 1.5 & nd & $0.34 \pm 0.06$ \\
\hline 19 & 1.8 & - & - & - & - & - & - & nd & 0.0 \\
\hline 20 & 0.0 & 0.0 & 1.3 & 0.06 & 0.24 & 2.4 & 0.7 & nd & $0.7 \pm 0.1$ \\
\hline 22 & 1.6 & 4.0 & 0.26 & 0.07 & 1.1 & 3.9 & 0.8 & nd & $2.8 \pm 0.4$ \\
\hline 24 & 0.0 & 3.35 & 0.03 & 0.23 & 0.63 & 1.8 & 0.5 & nd & $1.3 \pm 0.1$ \\
\hline 26 & 0.0 & 0.0 & 0.66 & 0.18 & 0.32 & 1.6 & 0.3 & nd & $0.29 \pm 0.04$ \\
\hline
\end{tabular}

${ }^{a}$ Normalized values of $\left|S_{h k l}\right|^{2}$ for the double diamond (D), plumber's nightmare (P), I-WP, and double gyroid (G) models were calculated using the parametric functions of Garstecki and Holyst. ${ }^{26}$ For the double gyroid $(\mathrm{G})$, double diamond (D), and plumber's nightmare (P) structures the volume of both networks was $18 \%$. For the I-WP structure, the volume of the 4 -fold network was $36 \%{ }^{b}$ The intensity of the $\{311\}$ and $\{421\}$ reflections of the D structure were below $0.1 \%$ of the intensity of the $\{211\}$ reflections. ${ }^{c}$ Elastic model of the double gyroid structure $\left(\mathrm{G}_{\mathrm{el}}\right)$ following $30 \%$ uniaxial contraction. The majority phase (PEO/aluminosilicate) was taken to be 10 times stiffer than the minority phase (PI), and Poisson's ratio for both phases was 0 . The PEO/ aluminosilicate had a density ${ }^{29}$ of $1.4 \mathrm{~g} / \mathrm{cm}^{3}$, while the PI domains ${ }^{49}$ had a density of $0.9 \mathrm{~g} / \mathrm{cm}^{3} \cdot{ }^{d}\left\langle\left|S_{h k l}\right|^{2}\right\rangle$ averaged for contraction along the [100], [110], [111], and $[16,9,4\}$ directions. ${ }^{e}$ Constant curvature model of double gyroid structure $\left(\mathrm{G}_{\mathrm{CC}}\right)$ under $30 \%$ uniaxial compression. ${ }^{f}$ Constant thickness model of double gyroid structure $\left(\mathrm{G}_{\mathrm{CT}}\right)$ under $30 \%$ uniaxial compression. ${ }^{g}$ Experimental values determined using pseudo-powder average as reported in Table 1. $h$ "-" indicates forbidden by crystallographic symmetry.

a

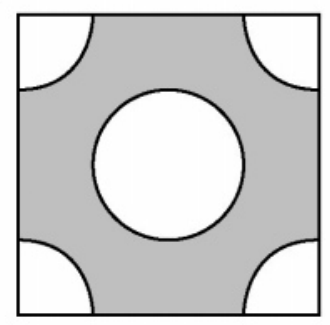

C

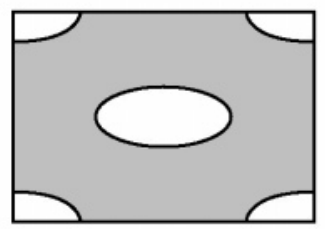

b

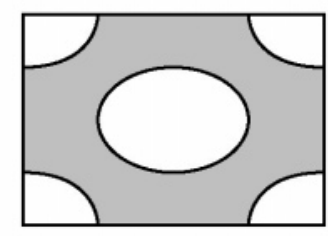

d

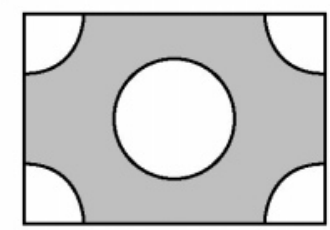

Figure 4. Structural change induced by uniaxial contraction. When the circular minority phase (white) and surrounding majority phase (gray) compress equally (b), the structure factors are essentially identical to those of the uncompressed structure (a). For an elastic material in which the majority phase is less compressible, the circular domains will preferentially flatten as shown in part c. For a liquid crystalline material, the energy of the interface between the majority and minority phase favors domains of constant thickness or constant curvature as shown in part $d$.

shows symmetry-forbidden reflections if the structure inside the unit cell is distorted relative to the crystal lattice.

Consequently, in some cases symmetry-forbidden reflections have been observed from block copolymers and mesoporous silica materials with compressed/stretched double gyroid (G) structures, while in other cases these reflections were not seen. For example, Sakurai and colleagues reported the appearance of $\{110\}$ and $\{200\}$ reflections after a double gyroid structure in a poly(styrene)- $b$-poly(butadiene)- $b$-poly(styrene) block copolymer was plastically deformed under tension. ${ }^{18}$ In contrast, a thin film of bicontinuous silica described by Hayward and colleagues $^{28}$ showed only the $\{211\}$ and $\{220\}$ reflections of

the Ia $\underline{3} d$ lattice despite a uniaxial compression of $\sim 15 \%$. In a more recent study ${ }^{19}$ of a double gyroid $(\mathrm{G})$ mesoporous silica film, the as-made structure did not show the forbidden $\{110\}$ or $\{200\}$ reflections ( $\sim 7 \%$ contraction). However, calcination caused the film to contract by $\sim 40 \%$ and weak $\{110\}$ reflections were then observed.

To see if a distorted double gyroid (distorted-G) structure is consistent with the structure factors of the material studied here, the structural deformations caused by lattice contraction were calculated for several models. The rheological properties of this block copolymer/aluminosilicate material varied during the solvent casting process. ${ }^{29}$ Initially, when the solvent content was high, the material should have been able to flow in response to applied stress. As the solvent content dropped, the PI and PEO/ aluminosilicate phases separated to form three-dimensional, interpenetrating networks. Even though the individual polymer blocks and aluminosilicate particles could still move within their respective domains, in this liquid crystalline state the domain topology could not readily change and the bulk material should have exhibited a solidlike response to applied stress. ${ }^{30}$ Finally, as the number of cross-links between aluminosilicate particles increased, the PEO and PI polymer blocks would have been immobilized by the three-dimensional network of covalent bonds within the PEO/aluminosilicate domains. In this heavily cross-linked state, the response of the material to applied stress should have been similar to that of an inhomogeneous, elastic solid.

The response of the heavily cross-linked state to an imposed strain was approximated using an elastic double gyroid $\left(\mathrm{G}_{\mathrm{el}}\right)$ model in which the PI and PEO/aluminosilicate domains were treated as solids with different elastic moduli. For the intermediate, liquid crystalline state, constant curvature ${ }^{31}\left(\mathrm{G}_{\mathrm{CC}}\right)$ and constant thickness ${ }^{9,13,20}\left(\mathrm{G}_{\mathrm{CT}}\right)$ structural models with the topology of the double gyroid structure were used to model the deformation of the PI and PEO/aluminosilicate domains. As illustrated in Figures $4 \mathrm{c}$ and $4 \mathrm{~d}$, contraction during these two stages of the solvent casting process should lead to different types of deformation. 
In an elastic, inhomogeneous material, the spatial dependence of the material's elastic properties causes the structure to deform relative to the crystal lattice. After a contraction, each point, $x_{k}$, moves to a new position, $X_{j}\left(x_{k}\right)$, given by

$$
X_{j}\left(x_{k}\right)=M_{j k} x_{k}+\sum_{m} A_{j}^{m} \exp \left(i q_{k}^{m} x_{k}\right)
$$

where $M_{j k}$ describes the bulk compression of the material and the change in structure within the unit cell is described by the Fourier amplitudes $A_{j}^{m}$ for reciprocal lattice vectors $q_{k}^{m}$. The resulting local strain is given by ${ }^{32}$

$$
e_{j k}\left(x_{l}\right)=\frac{1}{2}\left(\frac{\partial X_{j}}{\partial x_{k}}+\frac{\partial X_{k}}{\partial x_{j}}\right)-\delta_{j k}=\sum_{m} e_{j k}^{m} \exp \left(i q_{l}^{m} x_{l}\right)
$$

where

$$
e_{j k}^{m}=\frac{i}{2}\left(A_{j}^{m} q_{k}^{m}+A_{k}^{m} q_{j}^{m}\right)+\delta\left(q_{l}^{m}\right)\left(\frac{M_{j k}+M_{k j}}{2}-\delta_{j k}\right)
$$

Assuming the elastic response is locally isotropic, the stress field, $S_{j k}\left(x_{l}\right)$, is

$$
\begin{aligned}
S_{j k}\left(x_{l}\right) & =2 \mu\left(x_{l}\right) e_{j k}\left(x_{n}\right)+\lambda\left(x_{l}\right) e_{m m}\left(x_{n}\right) \delta_{j k} \\
& =\sum_{m, n}\left(2 \mu^{m} e_{j k}^{n}+\lambda^{m} e_{l l}^{n} \delta_{j k}\right) \exp \left(i q_{l}^{m} x_{l}+i q_{l}^{n} x_{l}\right)
\end{aligned}
$$

where $\mu\left(x_{l}\right)$ and $\lambda\left(x_{l}\right)$ are the first and second Lame elastic coefficients $^{32}$ at point $x_{l}$ and $\mu^{m}$ and $\lambda^{m}$ are the corresponding Fourier coefficients. The elastic energy per unit volume is then

$$
U_{\mathrm{e}}=\frac{1}{2} \sum_{m, n, p}\left(2 \mu^{m} e_{j k}^{n} e_{j k}^{p}+\lambda^{m} e_{j j}^{n} e_{k k}^{m}\right) \delta\left(q_{r}^{m}+q_{r}^{n}+q_{r}^{p}\right)
$$

The equilibrium structure can be rapidly determined by conjugate-gradient minimization ${ }^{33}$ of this elastic energy. The Fourier coefficients of the compressed structure are then given by

$$
\begin{aligned}
\rho^{\mathrm{c}, m} & =\int_{\mathrm{V}} \rho^{u}\left(x_{l}\right) \exp \left(-i q_{j}^{m} M_{j k}^{-1} X_{k}\left(x_{l}\right)\right) \frac{d^{3} x_{l}}{\operatorname{det}(M) V} \\
& \approx \frac{\rho^{\mathrm{u}, m}-i q_{j}^{m} \sum_{n, p} M_{j k}{ }^{-1} A_{k}^{p} \rho^{\mathrm{u}, n} \delta\left(q_{l}^{n}+q_{l}^{p}-q_{l}^{m}\right)}{\operatorname{det}(M)}
\end{aligned}
$$

where $\rho^{\mathrm{c}, m}$ and $\rho^{\mathrm{u}, m}$ are the Fourier coefficients for the compressed and uncompressed structures for the lattice vector $q_{j}^{m}$. Table 2 reports the structure factors for this elastic double gyroid $\left(\mathrm{G}_{\mathrm{el}}\right)$ model. Prior to contraction, the PEO/aluminosilicate domain was taken to be a constant-thickness membrane (volume $=64 \%$ ). Since the elastic properties of the domains change during the solvent-casting process, the full range of elastic moduli consistent with thermodynamics was tested. An upper bound on elastic deformation was estimated by taking the stiffness of the PEO/aluminosilicate phase to be 10 times that of the PI phase. As Poisson's ratio, $\sigma$, had a modest effect on structure factors, Table 2 only reports results for $\sigma=0$. Crystallites in the material had a variety of orientations relative to the strain direction so each structure factor, $\left\langle\left|S_{h k l}\right|^{2}\right\rangle$, was averaged over uniaxial contractions of $s=30 \%$ along the [100], [110], [111], and [16,9,4] directions. The $\{110\}$ and $\{200\}$ reflections for this elastic double gyroid $\left(\mathrm{G}_{\mathrm{el}}\right)$ model are considerably smaller than the measured values.
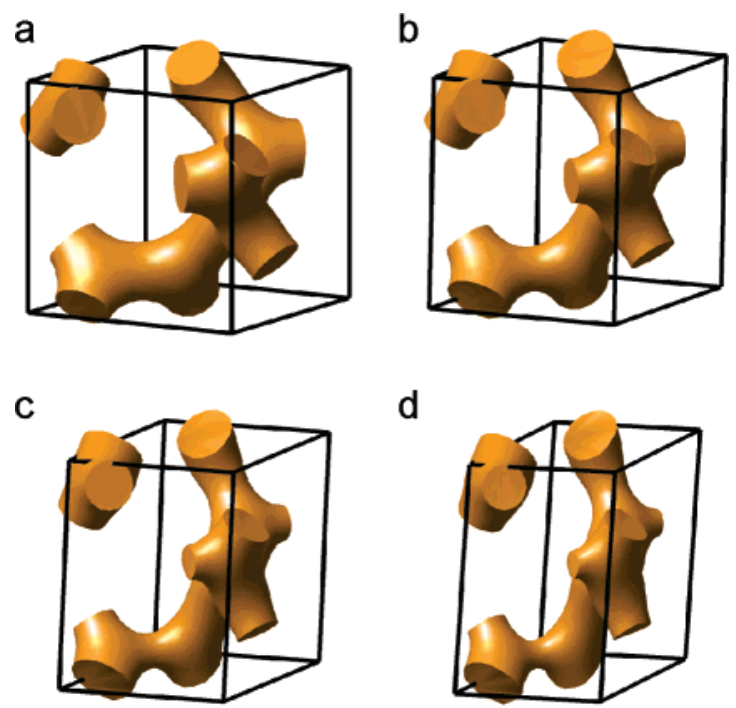

Figure 5. Deformation of the constant-curvature double gyroid $\left(\mathrm{G}_{\mathrm{CC}}\right)$ structure (18\% volume) undergoing (a) $0 \%$, (b) $10 \%$, (c) $20 \%$, and (d) $30 \%$ contraction along the $[16,9,4]$ direction. For clarity, only one of the two symmetry-related "single gyroid" networks is shown.

Because the major and minor domains form interpenetrating three-dimensional networks, the double gyroid $(\mathrm{G})$ structure can exhibit a solidlike response to applied stress ${ }^{30}$ even when the individual polymer blocks can move within their respective domains. During the intermediate stages of solvent casting, imposed strain could have caused relatively large changes in domain shape (but not topology) because the aluminosilicate particles and polymer chains were not fully immobilized by a network of covalent bonds within the PEO/aluminosilicate domains. The optimal domain shapes for a liquid crystal with a given unit cell and domain topology can be used to estimate the rearrangements during this stage of solvent casting. As the energetic interactions of such a hybrid/copolymer system have not been quantified, simpler energetic models were employed.

The shape of domain interfaces in block copolymers has previously been approximated using surfaces of constant curvature. ${ }^{31}$ Figure 5 illustrates the effects of compressing a single gyroid network bounded by a constant-curvature interface $\left(\mathrm{G}_{\mathrm{CC}}\right)$. As the lattice contracts, each 3-fold connector undergoes a distinctive distortion and the symmetries of the original network are clearly broken. For this calculation, the single gyroid network was described with a triangulated surface (2304 facets per unit cell) and the surface was then numerically optimized ${ }^{34,35}$ so as to achieve constant mean curvature under the constraint of a network volume of $18 \%$.

The Abbe transform ${ }^{13}$ was employed to compute structure factors for this single gyroid network. In the general triclinic lattice, the position of the double gyroid's second network could be ambiguous as the glide planes and screw axes of the $\mathrm{Q}^{230}$ lattice constrain its position in the cubic case. However, several different criteria for positioning the two networks yield indistinguishable structure factors. Figure 6 shows the average value of the $\{110\}$ and $\{200\}$ structure factors for this constantcurvature double gyroid $\left(\mathrm{G}_{\mathrm{CC}}\right)$ model as a function of contraction along the, [100], [110], [111], and [16,9,4] directions. The structure factors rapidly grow as the structure is compressed, and their average values for a contraction of $s=30 \%$ are reported in Table 2 .

Network structures in liquid crystals have also been described using a membrane of uniform thickness centered on an infinite 


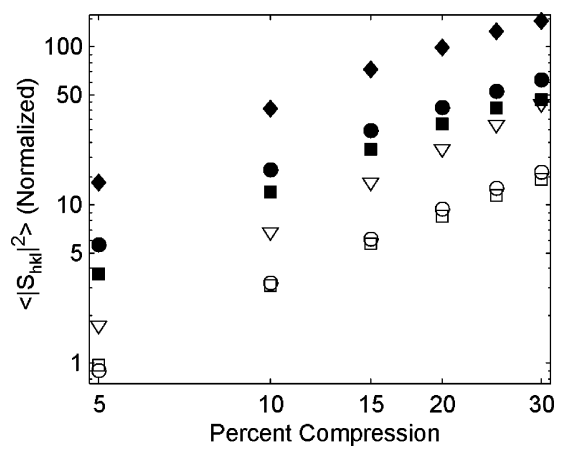

Figure 6. $\{110\}$ and $\{200\}$ structure factors of a constant curvature double gyroid $\left(\mathrm{G}_{\mathrm{CC}}\right)$ structure as a function of uniaxial contraction. Open/closed symbols correspond to the average $\{110\} /\{200\}$ structure factors for compression along the $[100](\diamond),[110](\square, \boldsymbol{D}),[111](\nabla)$ and $[16,9,4](\bigcirc, \bullet)$ directions. Structure factors are normalized so $\left|S_{211}\right|^{2}$ $=100$ in the uncompressed structure.
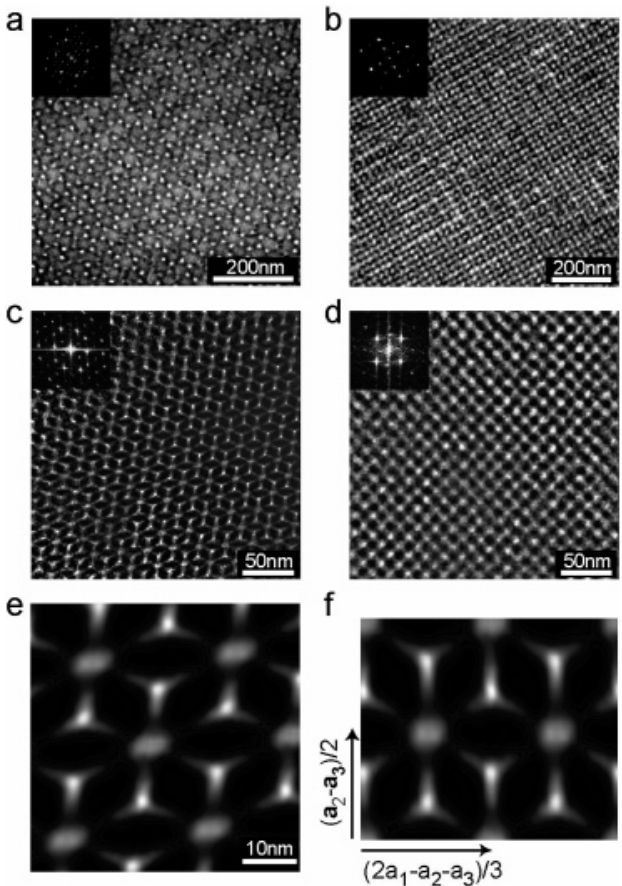

Figure 7. Bright-Field transmission electron micrographs (PEOaluminosilicate dark/PI or voids bright) of the as-made (top) and calcined material (bottom) highlighting the (a, c) 3-fold and (b, d) 4-fold projections of the cubic phase. The Fourier transform of each image is shown in the corresponding inset. Panel e shows an average of multiple unit cells of the [111] projection of the calcined material shown in panel c. Stretching the unit cell back to cubic (panel f) restores the hexagonal symmetry of the [111] projection. The arrows indicate the in-plane components of the cubic lattice vectors $\left(a_{1}, a_{2}, a_{3}\right)$.

periodic minimal surface (IPMS) ${ }^{9,13,20}$ where the thickness of the membrane determines its volume fraction. These "constantthickness" models are a good approximation for water-rich, surfactant bilayer network structures and have also been applied to the structure of block copolymers. ${ }^{13,26}$ For this constant thickness double gyroid $\left(\mathrm{G}_{\mathrm{CT}}\right)$ model, the midplane of the membrane was described with a discrete surface (1536 facets per unit cell) and the shape of this surface optimized numerically to achieve zero mean curvature for each facet. ${ }^{34,35}$ The thickness of the membrane was then adjusted to give a volume fraction of $64 \%$, and the positions of the inner and outer membrane surfaces were computed. Finally, structure factors were evaluated by applying Abbe's transformation to the discrete representation of the inner and outer membrane surfaces. ${ }^{13}$
Table 3. Unit Cell Parameters for Micrographs in Figure 7

\begin{tabular}{|c|c|c|c|}
\hline micrograph & $\begin{array}{l}\text { projected unit cell } \\
\text { vectors }(\mathrm{nm})\end{array}$ & $\begin{array}{c}\text { apparent } \\
\text { unit cell } \\
\text { size }(\mathrm{nm})\end{array}$ & $\begin{array}{c}\text { minimum } \\
\text { uniaxial } \\
\text { compression } \\
(\%)\end{array}$ \\
\hline as-made [111] & $a_{1}=45.2 x-6.3 y$ & & \\
\hline Figure $7 \mathrm{a}$ & $\begin{array}{l}a_{2}=-15.5 x+35.3 y \\
a_{3}=-29.6 x-28.9 y\end{array}$ & 56.2 & 18 \\
\hline as-made [100] & $a_{2}=63.8 x-16.5 y$ & 65.9 & 5 \\
\hline $\begin{array}{l}\text { Figure } 7 \mathrm{~b} \\
\text { calcined [111] }\end{array}$ & $\begin{array}{l}a_{3}=15.0 x+60.9 y \\
a_{1}=26.6 x+2.0 y\end{array}$ & & \\
\hline Figure $7 \mathrm{c}$ & $\begin{array}{l}a_{2}=-10.9 x+15.5 y \\
a_{3}=-15.7 x-17.5 y\end{array}$ & 33.4 & 33 \\
\hline calcined [100] & $a_{2}=36.3 x-1.4 y$ & 38.1 & 5 \\
\hline Figure $7 \mathrm{~d}$ & $a_{3}=0 x-37.7 y$ & & \\
\hline
\end{tabular}

Table 2 reports the structure factors for the constant thickness double gyroid $\left(\mathrm{G}_{\mathrm{CT}}\right)$ model after averaging over contractions of $s=30 \%$ along the [100], [110], [111], and [16,9,4] directions. As expected, larger structural rearrangements are possible when material can move within the continuous PI and PEO/aluminosilicate domains. Following a contraction of $s=30 \%$, the structure factors for the $\{110\}$ and $\{200\}$ reflections for both the constant curvature $\left(\mathrm{G}_{\mathrm{CC}}\right)$ and constant thickness $\left(\mathrm{G}_{\mathrm{CT}}\right)$ double gyroid models are considerably larger than the observed values. While neither the constant-thickness $\left(\mathrm{G}_{\mathrm{CT}}\right)$ nor the constantcurvature $\left(\mathrm{G}_{\mathrm{CC}}\right)$ models capture all the details of the copolymer/ aluminosilicate hybrid energetics, they confirm that large changes in structure factors can occur without changing the network topology. In summary, the calculations for the elastic $\left(\mathrm{G}_{\mathrm{el}}\right)$, constant-curvature $\left(\mathrm{G}_{\mathrm{CC}}\right)$ and constant-thickness $\left(\mathrm{G}_{\mathrm{CT}}\right)$ double gyroid models indicate that a double gyroid structure deformed by the solvent-casting process is consistent with the intensity of the observed $\{110\}$ and $\{200\}$ reflections.

Transmission Electron Microscopy. As reported previously, ${ }^{1,36}$ thin sections of the as-made and calcined materials were examined via bright-field TEM and representative micrographs are shown in Figure 7. Bright areas correspond to the minor phase (PI or voids) while dark areas correspond to the major phase (PEO/aluminosilicate). Despite some distortion of the lattice from solvent casting or sectioning, the classic fourfold ( [100] direction) and threefold ( [111] direction) orientations of a cubic lattice are still evident. The threefold "wagon-wheels" evident in Figure 7a,c are a common feature of cubic, bicontinuous structures. ${ }^{12}$

Following a uniaxial contraction, the projected lattice vectors $\left(a_{1}, a_{2}, a_{3}\right)$ of a cubic lattice are given by

$$
A_{p}=\left(\begin{array}{lll}
a_{1 x} & a_{2 x} & a_{3 x} \\
a_{1 y} & a_{2 y} & a_{3 y}
\end{array}\right)=d \times\left(\begin{array}{lll}
1 & 0 & 0 \\
0 & 1 & 0
\end{array}\right) \times\left(1-s \times n n^{T}\right) \times R
$$

where $d$ is the unit cell size, $s$ is the fractional contraction along an axis, $n$, and $R$ is a unitary $3 \times 3$ matrix specifying the orientation of the crystal axes. The unit cell size and minimum uniaxial contraction are then given by

$$
d=\sqrt{\lambda_{\text {big }}} \text { and } s \geq 1-\sqrt{\frac{\lambda_{\text {small }}}{\lambda_{\text {big }}}}
$$

where $\lambda_{\text {big }}$ and $\lambda_{\text {small }}$ are the larger and smaller eigenvalues of the square matrix, $A_{p} \times A_{p}{ }^{T}$.

The Fourier transform of each micrograph was used to determine its projected unit lattice vectors and the results are reported in Table 3. The average lattice sizes (as-made $d=61$ $\pm 5 \mathrm{~nm}$, calcined $d=36 \pm 3 \mathrm{~nm}$ ) agree with those obtained from SAXS (as-made $d=63.3 \pm 1.0 \mathrm{~nm}$, calcined $d=39.2 \pm$ 
a
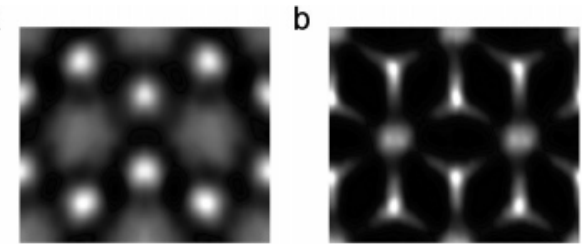

C

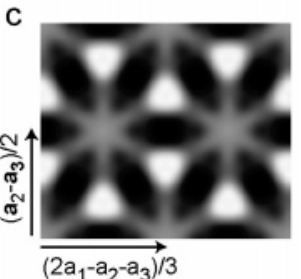

d

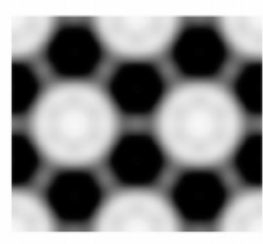

Figure 8. Averaged unit cells (rectified [111] projection, PEO/ aluminosilicate dark) for (a) as-made and (b) calcined material and corresponding projections of the (c) double gyroid (G) structure and (d) plumber's nightmare $(\mathrm{P})$ structure. The in-plane components of the cubic lattice vectors $\left(a_{1}, a_{2}, a_{3}\right)$ are indicated by arrows.
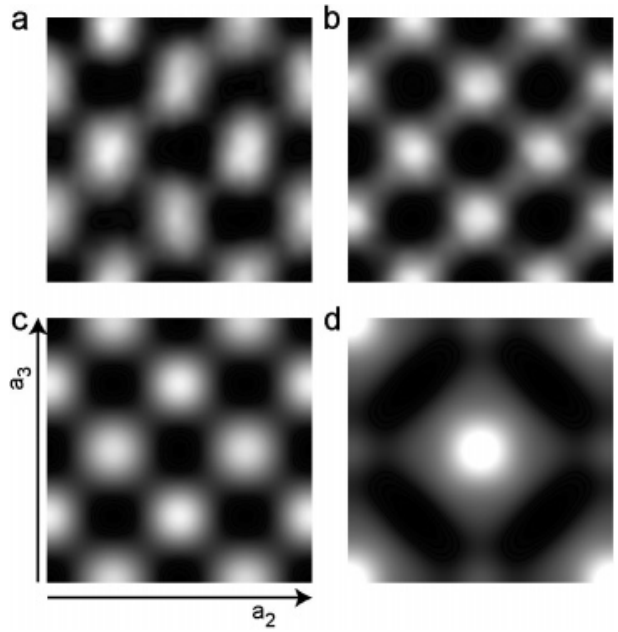

Figure 9. Averaged unit cells (rectified [100] projection, PEO/ aluminosilicate dark) for the (a) as-made and (b) calcined material and corresponding projections of the (c) double gyroid $(\mathrm{G})$ structure and (d) plumber's nightmare (P) structure. The [100] projection of both the double gyroid structure and plumber's nightmare structure form a square pattern of bright spots (PI phase), but the spots are twice as close in the double gyroid structure. Arrows mark the $a_{2}$ and $a_{3}$ cubic lattice vectors.

$0.8 \mathrm{~nm}$ ). As shown in Figures $7 \mathrm{e}$ and $7 \mathrm{f}$, an average unit cell could be obtained by summing an array of unit cells and then stretching this averaged structure back onto a cubic lattice. These "rectified" unit cells are displayed in Figures 8 and 9. To compare these images to structural models, projections of the network structures were evaluated by Fourier summation ${ }^{13}$ of structure factors obtained using the parametric equations of Garstecki and Holyst. ${ }^{26}$

The [111] projections of the as-made (Figure 8a) and calcined (Figure $8 \mathrm{~b}$ ) materials both show bright spots at the corners of the hexagonal cell. Contrast was better in the calcined material, and in the calcined unit cell the bright spots appear to be joined together at the center of the cell. The [111] projection of the double gyroid $(\mathrm{G})$ structure (Figure $8 \mathrm{c}$ ) has both of these features, but projections of the plumber's nightmare $(\mathrm{P})$ structure (Figure 8d) or I-WP structure do not. Although a projection of a specific half-unit cell of the double diamond (D) structure $^{13}$ looks similar to the double gyroid, the standard [111] projection of the full double diamond structure (Supporting Information Figure 1) also differs from the experimental micrographs.

The double gyroid structure can also be distinguished in the [100] projection. Although the [100] projections of all four cubic network structures (Supporting Information Figure 2) show a square grid of bright spots, ${ }^{14}$ the spots in the [100] projection of the double gyroid structure (Figure 9c) are twice as close together. In the [100] projections of the as-made (Figure 9a) and calcined (Figure 9b) material, the spot spacing matches the double gyroid structure.

\section{Discussion}

Of the network structures considered in this study, the SAXS and TEM data are best described by the distorted double gyroid structure. First, there are significant differences between the structure factors of the material and structure factors for models of plumber's nightmare (P), I-WP, and double diamond (D) networks. Modest deformations of these structures do not resolve these differences. In contrast, the observed structure factors are consistent with a double gyroid (G) structure deformed by uniaxial contraction. Second, TEMs of the material differ from [100] and [111] projections of the double diamond, plumber's nightmare and I-WP structures. Instead, the TEMs show the main features of [100] and [111] projections of the double gyroid structure. Thus, of all the above structures considered, the distorted double gyroid (distorted-G) structure is most consistent with the experimental data.

There are several reasons why the conclusions of this analysis differ from the conclusions of the original report. ${ }^{1,2}$ First, although the contraction of the material during solvent casting was described, the original analysis assumed the apparent symmetry of the structure was not altered by the contraction. On the basis of the present work, this assumption is reasonable when the contraction is not too large and/or the material deforms like an elastic solid. However, the large $(\sim 30 \%)$ contraction and possibility that the domains were relatively deformable in the early stages of solvent casting mean that for this material, symmetry-breaking effects need to be considered. Second, because the elliptical and oligo-crystalline character of the SAXS was difficult to analyze, quantitative estimates of the material's structure factors were not made in the original analysis. Finally, in the original analysis, TEM data were used primarily to establish the bicontinuous character of the structure and was compared to skeletal graphs ${ }^{2}$ rather than the projected density of structural models. Skeletal graphs illustrate the topology of bicontinuous structures but the corresponding TEM image can have quite a different appearance.

Because the double gyroid $(G)$ structure has numerous symmetry-forbidden reflections, the identification or exclusion of the double gyroid structure is particularly complicated by symmetry-breaking effects. The present work illustrates the advantages of directly comparing experimental SAXS and TEM data with predictions for a proposed structure. In the past, this was challenging for morphologies like the double gyroid $(\mathrm{G})$ because of their complex three-dimensional structures. However, using a level set description ${ }^{48}$ and modern scientific programming environments (Matlab, Mathematica, IDL, etc.), it is now straightforward to visualize complex structures and calculate the corresponding SAXS and TEM projections. ${ }^{19}$ Furthermore, with this approach it is easy to examine variations to structures, such as deformations of the double gyroid. By comparing the experimental data to predictions for a given structure, consistency can be directly established.

The original identification of a plumber's nightmare (P) structure in this material was surprising since the double gyroid (G) structure forms in the parent PI- $b$-PEO copolymer system. ${ }^{37}$ 
In bicontinuous block copolymer structures, the minority domain occupies the channels and nodes of the network. The entropic penalty for stretching the chains of the minority block to the center of each node favors the double gyroid structure (3-fold nodes) over the double diamond (4-fold nodes) and plumber's nightmare structures (6-fold nodes). ${ }^{38}$ Consequently, for pure block copolymers only 3-fold networks have been predicted ${ }^{39-41}$ or observed. ${ }^{5,42-44}$ Chain stretching in a 4-fold or 6-fold node can be reduced by the presence of a second component that occupies the center of the node. For example, adding homopolymer to the minority phase of a diblock copolymer is predicted to stabilize the double diamond morphology. ${ }^{38}$ The ceramic component in a block copolymer-ceramic material may act in a similar fashion and nodes with more than three arms were observed in a PI- $b$-PEO/aluminosilicate composite in which the $\mathrm{PEO} /$ aluminosilicate phase occupied the channels of a network structure. ${ }^{45}$ Also in a calcined PI- $b$-PEO/aluminosilicate material with a PEO/aluminosilicate minority volume fraction of 0.36 , SAXS was clearly inconsistent with a gyroid. ${ }^{46}$ In contrast, in the material described in the present study, the aluminosilicate sol particles and PEO block form the membrane separating the two PI networks of channels and nodes. Since the aluminosilicate particles cannot directly relieve frustration in the PI nodes, a structure with 3-fold nodes should be favored, in agreement with the reanalysis of the material's structure.

Finally, the present work relies on structural models to interpret the experimental data. The selection of these models introduces implicit assumptions about the symmetry and structure of the material. Furthermore, even if a given model is consistent with all the experimental data, there is no guarantee it is unique. Methods that directly determine the structure of a material, such as 3-D electron tomography, ${ }^{11,47}$ avoid many of these difficulties. Since this PI- $b$-PEO/aluminosilicate material is well suited to 3-D electron tomography, it would be very interesting to study the material with this technique in the future.

\section{Conclusion}

The solvent-casting process used to prepare this material caused the cubic, bicontinuous network structure within the material to undergo a uniaxial contraction of $\sim 30 \%$. Some of the symmetries of the original cubic structure could have been broken by this contraction and so the observed $\{110\}$ and $\{200\}$ reflections do not exclude the double gyroid $(G)$ structure. SAXS from the material is consistent with structure factors for constantcurvature and constant-thickness models of a distorted double gyroid (distorted-G) structure. Furthermore, [100] and [111] projections of the double gyroid structure match the TEM data. Thus, a distorted version of the double gyroid $(\mathrm{G})$ morphology is consistent with SAXS and TEM data from this material.

Acknowledgment. We thank Marleen Kamperman for helpful comments on the manuscript and U.W. thanks Osamu Terasaki for fruitful discussions. The work was supported by National Science Foundation (NSF) Grants DMR-0072009 and DMR-0605856, Department of Energy Grant DE-FG0297ER62443, the Cornell Center for Materials Research, a Materials Research Science and Engineering Center of the National Science Foundation (DMR-0079992). The experiments made use of the Cornell High-Energy Synchrotron Source (CHESS), supported by the NSF and the NIH-National Institute of General Medical Sciences under Award DMR-0225180.

Supporting Information Available: [100] and [111] projections for the double gyroid $(\mathrm{G})$, double-diamond (D), plumber's night- mare (P), and I-WP morphologies. This material is available free of charge via the Internet at http://pubs.acs.org.

\section{References and Notes}

(1) Finnefrock, A. C.; Ulrich, R.; Du Chesne, A.; Honeker, C. C.; Schumacher, K.; Unger, K. K.; Gruner, S. M.; Wiesner, U. Angew. Chem., Int. Ed. 2001, 40, 1207-1211.

(2) Finnefrock, A. C.; Ulrich, R.; Toombes, G. E. S.; Gruner, S. M.; Wiesner, U. J. Am. Chem. Soc. 2003, 125, 13084-13093.

(3) Huse, D. A.; Leibler, S. J. Phys. France 1988, 49, 605-620.

(4) Schoen, A. H. Infinite Periodic Minimal Surfaces without SelfIntersections; NASA Technical Note, TN D-5541; NASA: Washington, DC, 1970

(5) Hajduk, D. A.; Harper, P. E.; Gruner, S. M.; Honeker, C. C.; Kim, G.; Thomas, E. L.; Fetters, L. J. Macromolecules 1994, 27, 40634075 .

(6) Schwarz, H. A. Gesammelte Mathematische Abhandlungen, Springer: Berlin, Germany, 1890.

(7) Gao, C.; Sakamoto, Y.; Sakamoto, K.; Terasaki, O.; Che, S. Angew. Chem., Int. Ed. 2006, 45, 4295-4298.

(8) Radiman, S.; Toprakcioglu, C.; Faruqi, A. R. J. Phys. France 1990 51, 1501-1508

(9) Hyde, S. T. Curr. Opin. Solid State Mater. Sci. 1996, 1, 653-662.

(10) Anderson, D.; Wennerstrom, H.; Olsson, U. J. Chem. Phys. 1989, 93, 4243-4253.

(11) Jinnai, H.; Hasegawa, H.; Nishikawa, Y.; Sevink, G.; Braunfeld, M.; Agard, D.; Spontak, R. Macromol. Rapid Commun. 2006, 27, 14241429.

(12) Hajduk, D. A.; Harper, P. E.; Gruner, S. M.; Honeker, C. C.; Kim, G.; Thomas, E. L.; Fetters, L. J. Macromolecules 1995, 28, 25072573.

(13) Harper, P. E. Ph.D. Thesis, Princeton University, Princeton, NJ, 1996.

(14) Benedicto, A. D.; O'Brien, D. F. Macromolecules 1997, 30, 33953402 .

(15) Matsen, M. W. J. Chem. Phys. 1998, 108, 785-796.

(16) Matsushita, Y.; Suzuki, J.; Seki, M. Physica B 1998, 248, 238-242.

(17) International Tables for Crystallography; Hahn, T., Ed.; Kluwer Academic Publishers: Boston, MA, 2002, Vol. A: Space-Group Symmetry.

(18) Sakurai, S.; Isobe, D.; Okamoto, S.; Yao, T.; Nomura, S. Phys. Rev. E: Stat., Nonlinear, Soft Matter Phys. 2001, 63, 061803.

(19) Urade, V. N.; Wei, T. C.; Tate, M. P.; Kowalski, J. D.; Hillhouse, H. W. Chem. Mater. 2007, 19, 768-777.

(20) Anderson, D. M.; Gruner, S. M.; Leibler, S. Proc. Natl. Acad. Sci. U.S.A. 1988, 85, 5364-5368

(21) Klotz, M.; Albouy, P.; Ayral, A.; Menager, C.; Grosso, D.; Van der Lee, A.; Cabuil, V.; Babonneau, F.; Guizard, C. Chem. Mater. 2000, 12, 1721-1728

(22) Young, R. A.; Wiles, D. B. J. Appl. Crystallogr. 1982, 15, 430-438.

(23) Humlicek, J. J. Quant. Spectrosc. Radiat. Transfer 1982, 27, 437444.

(24) Monnier, A.; Schuth, F.; Huo, Q.; Kumar, D.; Marolese, D.; Maxwell, R. S.; Stucky, G. D.; Krishnamurty, M.; Petroff, P.; Firouzi, A.; Janicke, M.; Chmelka, B. F. Science (Washington, DC, U.S.) 1993 261, 1299-1303.

(25) Sakamoto, Y.; Kaneda, M.; Terasaki, O.; Zhao, D. Y.; Kim, J. M.; Stucky, G.; Shin, H.; Ryoo, R. Nature (London) 2000, 408, 449453.

(26) Garstecki, P.; Holyst, R. Macromolecules 2003, 36, 9181-9190.

(27) Garstecki, P.; Holyst, R. Macromolecules 2003, 36, 9191-9198.

(28) Hayward, R. C.; Alberius, P. C. A.; Kramer, E. J.; Chmelka, B. F. Langmuir 2004, 20, 5998-6004.

(29) Jain, A.; Wiesner, U. Macromolecules 2004, 37, 5665-5670.

(30) Kossuth, M. B.; Morse, D. C.; Bates, F. S. J. Rheol. 1999, 43, 167196.

(31) Thomas, E. L.; Anderson, D. M.; Henkee, C. S.; Hoffman, D. Nature (London) 1988, 334, 598-601.

(32) Feynman, R. P.; Leighton, R. B.; Sands, M. The Feynman Lectures on Physics; Addison-Wesley: Sydney, Australia, 1977, Vol. 2, p 39 6.

(33) Press, W.; Teukolsky, S. A.; Vetterling, W. T.; Flannery, B. P. Numerical Recipes: The Art of Scientific Computing; Cambridge University Press: New York, 1986.

(34) Brakke, K. Exp. Math. 1992, 1, 141-165.

(35) Brakke, K. A. Philos. Trans. R. Soc. London, Ser. A 1996, 354, $2143-$ 2157.

(36) Ulrich, R. Ph.D. Thesis, Johannes-Gutenberg University, Mainz, Germany, 2000.

(37) Floudas, G.; Vazaiou, B.; Schipper, F.; Ulrich, R.; Wiesner, U.; Iatrou, H.; Hadjichristidis, N. Macromolecules 2001, 34, 2947-2957. 
(38) Matsen, M. W.; Bates, F. S. Macromolecules 1996, 29, 76417644.

(39) Matsen, M. W.; Schick, M. Phys. Rev. Lett. 1994, 72, 26602663.

(40) Tyler, C.; Morse, D. Phys. Rev. Lett. 2005, 94, 208302.

(41) Cochran, E.; Garcia-Cervera, C.; Fredrickson, G. H. Macromolecules 2006, 39, 2449-2451.

(42) Bailey, T. S.; Hardy, C. M.; Epps, T. H., III; Bates, F. S. Macromolecules 2002, 35, 7007-7017.

(43) Cochran, E.; Bates, F. S. Phys. Rev. Lett. 2004, 93, 087802-1.

(44) Takenaka, M.; Wakada, T.; Akasaka, S.; Nishitusi, S.; Saijo, K.; Shimizu, H.; Kim, M. I.; Hasegawa, H. Macromolecules, 2007, 40, 4399-4402.
(45) Warren, S. C.; DiSalvo, F. J.; Wiesner, U. Nat. Mater. 2007, 6, 156161.

(46) Jain, A.; Toombes, G. E. S.; Hall, L. M.; Mahajan, S.; Garcia, C. B. W.; Probst, W.; Gruner, S. M.; Wiesner, U. Angew. Chem., Int. Ed. 2005, 44, 1226-1229.

(47) Spontak, R. J.; Fung, J. C.; Braunfeld, M. B.; Sedat, J. W.; Agard, D. A.; Kane, L.; Smith, S. D.; Satkowski, M. M.; Ashraf, A.; Hajduk, D. A.; Gruner, S. M. Macromolecules 1996, 29, 4494-4507.

(48) Wohlgemuth, M.; Yufa, N.; Hoffman, J.; Thomas, E. Macromolecules 2001, 34, 6083-6089.

(49) Fetters, L. J.; Lohse, D. J.; Richter, D.; Witten, T. A.; Zirkel, A. Macromolecules 1994, 27, 4639-4647.

MA0715369 